\title{
The Strange Eigenmode in Lagrangian Coordinates
}

\author{
Jean-Luc Thiffeault* \\ Department of Mathematics, Imperial College London, SW7 2AZ, United Kingdom
}

(Dated: June 5, 2018)

\begin{abstract}
For a distribution advected by a simple chaotic map with diffusion, the "strange eigenmode" is investigated from the Lagrangian (material) viewpoint and compared to its Eulerian (spatial) counterpart. The eigenmode embodies the balance between diffusion and exponential stretching by a chaotic flow. It is not strictly an eigenmode in Lagrangian coordinates, because its spectrum is rescaled exponentially rapidly.
\end{abstract}

There are two main types of coordinates used to represent fluid flow and dynamical systems. Eulerian (or spatial) coordinates are fixed in space, while Lagrangian (or material) coordinates follow parcels of fluid. Strange eigenmodes are persistent patterns in mixing - they can decay slowly, and hence remain visible in the concentration field for a long time. So far, these have been studied from the Eulerian viewpoint. Here we describe the nature of the strange eigenmode in Lagrangian coordinates for a simple map. It is not a true eigenmode because its wavelength is continuously rescaled in time.

\section{INTRODUCTION}

The enhanced mixing of a passive scalar is one of the most direct consequences of chaos: a flow whose trajectories exhibit sensitivity to initial conditions will lead to rapid mixing. There are powerful theories based on the distribution of Lyapunov exponents [1-3] that link the mixing rate of the passive scalar with the chaotic properties of the flow. It has been recently suggested, following earlier work of Pierrehumbert [4-10], that the mixing properties of the flow can often be elucidated only by solving a full eigenvalue problem for the advection-diffusion operator, in an analogous manner to what is done for the kinematic dynamo [11]. The resulting eigenfunctions have been dubbed strange eigenmodes by Pierrehumbert, and are closely related to Pollicott-Ruelle resonances in ergodic theory [12-14], which describe the long-time decay of correlations in mixing hyperbolic dynamical systems. Strange eigenmodes have also been observed experimentally [15, 16]. They are often called persistent patterns or large-scale eigenfunctions.

The strange eigenmodes reflect a balance between advection and diffusion. On its own, advection is incapable of achieving mixing: it shuffles the concentration field of the passive scalar but does not decrease its fluctuations. The role of advection is to stir the concentration field, thereby creating sharp gradients in concentration. Physically, these gradients are reflected in the filamentation experienced by a blob of dye when it is stirred. The sharp gradients enhance the role of diffusion tremendously, and this allows mixing to proceed. As the diffusivity of the scalar is made smaller, the scale at which this mixing occurs decreases, so the concentration field appears very rough. In the limit of arbitrarily small diffusivity, the concentration field is not smooth: it consists of a superposition of strange eigenmodes. The

${ }^{*}$ Electronic address: jeanluc@imperial.ac.uk 
dominant one among these eigenfunctions is called the strange eigenmode, although there may be several of comparable importance.

In the present work we tie the two types of theories together (i.e., Lyapunov exponentbased and strange eigenmode) for a specific system, which was already studied in [8] from the strange eigenmode viewpoint. The strange eigenmode represents a fundamentally Eulerian (spatial) view of mixing, whereas the Lyapunov exponent view is Lagrangian (material), following as it does the stretching history of fluid elements. In the strongly chaotic systems we deal with in the present context, Lagrangian and Eulerian coordinates are related by a convoluted transformation. This transformation is so complex that its specific form is inaccessible (even numerically) after some time, a reflection of sensitivity to initial conditions. For long times, the two frames must be regarded as essentially independent: we cannot simply solve a problem in Eulerian coordinates and transform to Lagrangian coordinates (or vice-versa). Thus we believe it is worthwhile to take a chaotic system whose solution has already been obtained in Eulerian coordinates and solve it in Lagrangian coordinates. As indicated above, this links two views of mixing together, and in particular illustrates the nature of strange eigenmodes in Lagrangian coordinates. This also indicates the source of the breakdown of local theories. We will show that there exists a kind of Lagrangian strange eigenmode, which is not quite an eigenmode but which exhibits similar features: specifically, it is an eigenmode if an appropriate time-dependent rescaling of coordinates is performed (exponential in time). This rescaling is closely related to the "cone" involved in diffusive problems in the presence of an exponentially stretching flow [17]. We call it the cone of safety, because modes inside it are sheltered from diffusion at a given time.

We introduce the system to be studied, the perturbed cat map, in Section II. We find its finite-time Lyapunov exponents and eigenvectors using first-order perturbation theory. In Section III we partially solve the advection-diffusion equation for our map, again using perturbation theory. Some numerical work is needed to complete the solution, and this is described in Section IV. Finally, a few concluding remarks are offered in Section V.

\section{THE PERTURBED CAT MAP}

The strange eigenmode has for the most part been studied in maps, because these present great advantages for analytical work. This is also reasonable since experimental work has so far focused on time-periodic flows $[15,16]$. As in [8], we consider the map

$$
\mathcal{M}(\boldsymbol{x})=\mathbb{M} \cdot \boldsymbol{x}+\boldsymbol{\phi}(\boldsymbol{x}),
$$

defined on the unit two-torus, $\mathcal{T}^{2}=[0,1]^{2}$. Here $\mathbb{M}$ is a matrix of integers with unit determinant, and $\phi(\boldsymbol{x})$ is a doubly-periodic function, so that $\mathcal{M}$ is a diffeomorphism; specifically, we take

$$
\mathbb{M}=\left(\begin{array}{ll}
2 & 1 \\
1 & 1
\end{array}\right) ; \quad \phi(\boldsymbol{x})=\frac{K}{2 \pi}\left(\begin{array}{c}
\sin 2 \pi x_{1} \\
\sin 2 \pi x_{1}
\end{array}\right) ;
$$

where $\boldsymbol{\phi}(\boldsymbol{x})$ is chosen such that $\mathcal{M}$ is area-preserving. For $K=0,(2.1)$ is the usual cat map of Arnold [18]. The map (2.1) inherits much of the simplicity of the cat map, but the perturbation allows for more complex - and less singular - behavior. The action of the map is depicted in Fig. 1. For small $K$, the map is very close to the cat map, but the implications of the perturbation for mixing are profound, as we will now discuss.

We are interested in the mixing properties of the map $\mathcal{M}$. In Ref. [8] mixing in this map was investigated from an Eulerian perspective: advection alternated with diffusion and 


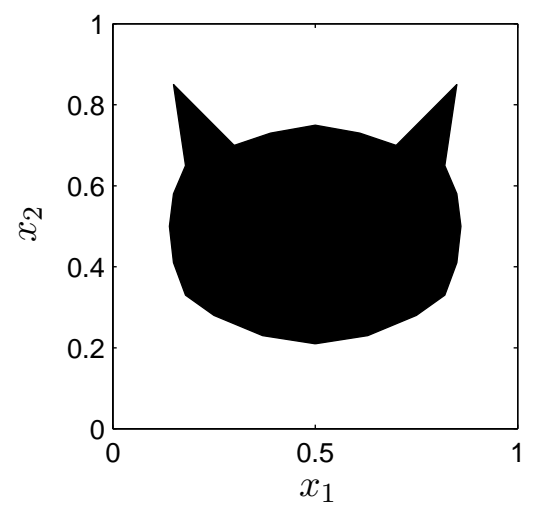

(a)

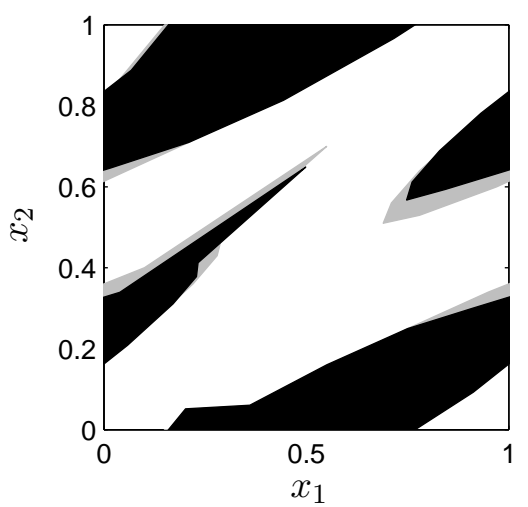

(b)
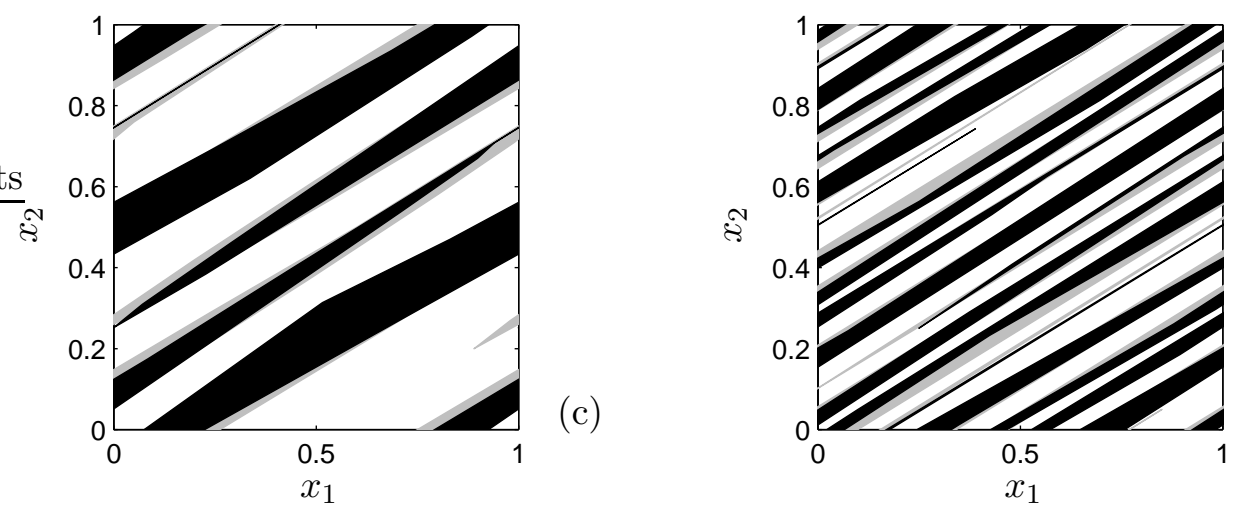

(d)

FIG. 1: Action of the perturbed cat map for $K=0.4$. (a) Initial pattern; (b) First iterate; (c) Second iterate; (d) Third iterate. The gray shading shows the action of the unperturbed cat map.

the central object was the distribution of the concentration field in Eulerian coordinates. For $K=0$, mixing occurs superexponentially in the map, due to the lack of dispersion in Fourier space. The concentration in a given Fourier mode is mapped entirely to one mode of higher wavenumber, and so on to ever higher wavenumbers. This sequence of wavenumbers have exponentially-growing magnitudes. Because diffusion is exponential in the wavenumber, the net result is superexponential decay (i.e., the exponential of minus an exponential in time).

For $K \neq 0$, the situation is radically different. The map now disperses concentration among many Fourier modes at each iteration. In particular, some concentration is always mapped back to the lowest allowable wavenumber (the grave mode), on which the weak diffusion is ineffective. The decay of the scalar is then limited by how much concentration is mapped to this grave mode at each iteration. The grave mode thus forms the seed of a strange eigenmode, since an eigenmode is by definition a recurring feature. The concentration field thus settles into the slowest-decaying eigenfunction of the advection-diffusion operator-the strange eigenmode - analogously to earlier work [4-7, 9, 10].

Here we wish to solve the same problem as in Ref. [8], but in Lagrangian coordinates. This means that we focus on following fluid elements and describing how they deform under the action of the map. To solve the advection-diffusion problem in Lagrangian coordinates, it is necessary to have expressions for the finite-time Lyapunov exponents of the map [19] (or equivalently the coefficients of expansion) and their associated characteristic directions, as a function of Lagrangian coordinates, and not just their distribution (we will see why this 
is so in Section III). Because the finite-time Lyapunov exponents are easily derived for the cat map $(K=0)$, we shall proceed perturbatively, assuming $K$ is small.

First let us give the Lyapunov exponents and associated characteristic directions for the unperturbed cat map. The Lyapunov exponents are the logarithms of the eigenvalues of $\mathbb{M}$, and the characteristic directions are the corresponding eigenvectors. It is convenient to introduce an angle $\theta$ in terms of which the eigenvectors of $\mathbb{M}$ are

$$
\left(\begin{array}{ll}
\hat{\mathbf{u}} & \hat{\mathbf{s}}
\end{array}\right)=\left(\begin{array}{cc}
\cos \theta & -\sin \theta \\
\sin \theta & \cos \theta
\end{array}\right)
$$

with $\cos ^{2} \theta=\frac{1}{2}(1+1 / \sqrt{5})$ and $\sin ^{2} \theta=\frac{1}{2}(1-1 / \sqrt{5})$. Then the corresponding eigenvalues of $\mathbb{M}$ are

$$
\begin{gathered}
\Lambda_{\mathrm{u}}=\Lambda=\frac{1}{2}(3+\sqrt{5})=1+\cot \theta=\cot ^{2} \theta, \\
\Lambda_{\mathrm{s}}=\Lambda^{-1}=\frac{1}{2}(3-\sqrt{5})=1-\tan \theta=\tan ^{2} \theta .
\end{gathered}
$$

These equalities are specific to this particular angle, as is the relation $\tan \theta=\cot \theta-1$. The $\hat{\mathbf{u}}$ direction is associated with stretching, and $\hat{\mathbf{s}}$ with contraction.

The coefficients of expansion (given by $\Lambda^{n}$ and $\Lambda^{-n}$ after $n$ iterations of the map) and characteristic directions for the linear cat map are uniform in space. Now we derive their value for $K$ nonzero but small, using perturbation theory. The problem is to find the eigenvalues and eigenvectors of the matrix $g^{(n)}$, with components

$$
g_{p q}^{(n)}:=\sum_{m} \frac{\partial x_{m}^{(n)}}{\partial X_{p}} \frac{\partial x_{m}^{(n)}}{\partial X_{q}},
$$

often called the metric tensor (or Cauchy-Green strain tensor in fluid mechanics). Here $\boldsymbol{X}$ is the Lagrangian label (coordinate) and $\boldsymbol{x}=\boldsymbol{x}^{(n)}(\boldsymbol{X})$ is the $n$th iterate of the point $\boldsymbol{X}$ under the action of the map $(2.1)$ (so that $\boldsymbol{x}^{(0)}(\boldsymbol{X})=\boldsymbol{X}$ ). The metric tensor describes the stretching experienced at the $n$th iteration by a fluid element initially at $\boldsymbol{X}$. Its eigenvalues and eigenvectors give the shape and orientation at the $n$th iteration of an ellipsoid representing an initially spherical infinitesimal element of fluid.

Before we can apply perturbation theory to the metric tensor, we must find the form of the perturbation itself. To first order in $K$, the $n$th iterate of the map (2.1) is

$$
\boldsymbol{x}^{(n)}=\mathcal{M}^{n}(\boldsymbol{X})=\mathbb{M}^{n} \cdot \boldsymbol{X}+\sum_{m=0}^{n-1} \mathbb{M}^{m} \cdot \boldsymbol{\phi}\left(\mathbb{M}^{n-m-1} \boldsymbol{X}\right) .
$$

The Jacobian matrix of this transformation is

$$
\frac{\partial \boldsymbol{x}^{(n)}}{\partial \boldsymbol{X}}=\mathbb{M}^{n}+\sum_{m=0}^{n-1} \mathbb{M}^{m} \cdot \frac{\partial \boldsymbol{\phi}}{\partial \boldsymbol{x}}\left(\mathbb{M}^{n-m-1} \boldsymbol{X}\right) \cdot \mathbb{M}^{n-m-1}
$$

where the numerator corresponds to rows and the denominator to columns of a matrix. We must now construct the metric tensor (2.5), which to leading order in $K$ is

$$
g_{K}^{(n)}=\widetilde{\mathbb{M}}^{n} \mathbb{M}^{n}+\left\{h^{(n)}+\widetilde{h^{(n)}}\right\}
$$


with

$$
h^{(n)}:=\widetilde{\mathbb{M}}^{n} \cdot \sum_{m=0}^{n-1} \mathbb{M}^{m} \cdot \frac{\partial \phi}{\partial \boldsymbol{x}}\left(\mathbb{M}^{n-m-1} \boldsymbol{X}\right) \cdot \mathbb{M}^{n-m-1}
$$

and the tilde denotes the transpose of a matrix. The unperturbed metric is $g_{0}^{(n)}=\widetilde{\mathbb{M}}^{n} \mathbb{M}^{n}$, with eigenvalues $\Lambda^{2 n}$ and $\Lambda^{-2 n}$ and eigenvectors given by (2.3). The perturbation is the bracketed term in (2.8). Finding the eigenvalues and eigenvectors of the symmetric matrix $g^{(n)}$ to leading order in $K$ is a straightforward application of perturbation theory for symmetric matrices, familiar from quantum mechanics. For more details we refer the reader to standard texts on the subject $[20,21]$.

To leading order in $K$, the coefficient of stretching is written as

$$
\Lambda_{K}^{(n)}(\boldsymbol{X})=\Lambda^{n}\left(1+K \eta^{(n)}(\boldsymbol{X})\right)
$$

where $\Lambda^{n}$ is the coefficient of stretching of the unperturbed cat map, and the correction $\Lambda^{n} K \eta^{(n)}(\boldsymbol{X})$ is obtained from the perturbation by contraction with the unperturbed eigenvectors $[20,21]$,

$$
\Lambda^{n} K \eta^{(n)}(\boldsymbol{X})=\frac{1}{2} \hat{\mathbf{u}} \cdot\left\{h^{(n)}+\widetilde{h^{(n)}}\right\} \cdot \hat{\mathbf{u}}=\hat{\mathbf{u}} \cdot h^{(n)} \cdot \hat{\mathbf{u}} .
$$

Observe that because the coefficient of stretching is the square root of the largest eigenvalue of $g_{K}^{(n)}$, there is an extra factor of $1 / 2$ to leading order in $K$. Using the fact that, for $\mathbb{M}$ symmetric, $\mathbb{M} \cdot \hat{\mathbf{u}}=\hat{\mathbf{u}} \cdot \mathbb{M}=\Lambda \hat{\mathbf{u}}$, we find Eqs. (2.9) and (2.11) give

$$
\eta^{(n)}=\sin \theta \cos \theta \sum_{m=0}^{n-1} \cos \left(2 \pi\left(\mathbb{M}^{m} \cdot \boldsymbol{X}\right)_{1}\right)
$$

where we have substituted the specific form of the map, given by (2.2). The subscript ' 1 ' in (2.12) indicates the $x_{1}$ component of a vector.

The perturbed eigenvectors can be written as

$$
\hat{\mathbf{u}}_{K}^{(n)}(\boldsymbol{X})=\hat{\mathbf{u}}+K \zeta^{(n)}(\boldsymbol{X}) \hat{\mathbf{s}}, \quad \hat{\mathbf{s}}_{K}^{(n)}(\boldsymbol{X})=\hat{\mathbf{s}}-K \zeta^{(n)}(\boldsymbol{X}) \hat{\mathbf{u}},
$$

where $K \zeta^{(n)}$ may be regarded as a small angle of rotation. Again we follow standard matrix perturbation theory $[20,21]$, so that the angle of rotation is given by

$$
K \zeta^{(n)}(\boldsymbol{X})=\frac{\hat{\mathbf{u}} \cdot\left\{h^{(n)}+\widetilde{h^{(n)}}\right\} \cdot \hat{\mathbf{s}}}{\Lambda^{2 n}-\Lambda^{-2 n}},
$$

which after some reduction and the use of (2.2) yields

$$
\zeta^{(n)}=\frac{1}{\Lambda^{2 n}-\Lambda^{-2 n}}\left(\zeta_{+}^{(n)}+\zeta_{-}^{(n)}\right)
$$

with

$$
\zeta_{ \pm}^{(n)}:=\frac{1}{2}(\cos 2 \theta \mp 1) \sum_{m=0}^{n-1} \Lambda^{ \pm 2(n-m)} \cos \left(2 \pi\left(\mathbb{M}^{m} \cdot \boldsymbol{X}\right)_{1}\right)
$$




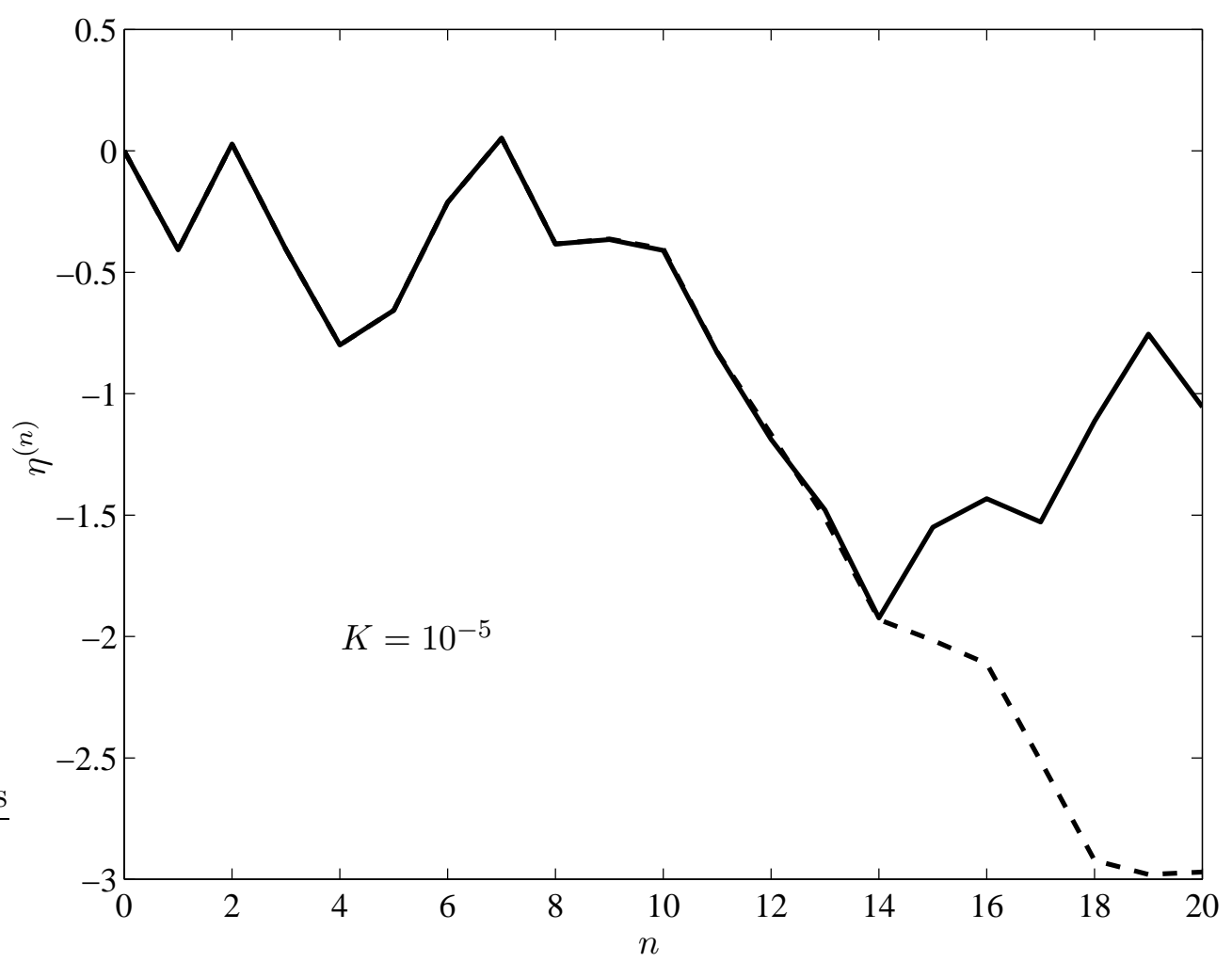

FIG. 2: Numerical solution (solid) and first-order solution $\eta^{(n)}$ (dashed) from Eq. (2.12) for $K=10^{-5}$. The solutions diverge after several iterations because we are perturbing off a chaotic trajectory.

Note that the asymptotic direction $(n \gg 1)$ is dominated by $\zeta_{+}^{(n)}$, so that

$$
\zeta^{(n)} \simeq \cos ^{2} \theta \sum_{m=0}^{n-1} \Lambda^{-2 m} \cos \left(2 \pi\left(\mathbb{M}^{m} \cdot \boldsymbol{X}\right)_{1}\right), \quad n \gg 1 .
$$

In this form it is easy to check that $\hat{\mathbf{u}} \cdot \nabla \zeta^{(n)}=\hat{\mathbf{s}} \cdot \nabla \eta^{(n)}$ for $n \gg 1$, as required by the differential constraint $\nabla \cdot \hat{\mathbf{s}}_{K}^{(n)}+\hat{\mathbf{s}}_{K}^{(n)} \cdot \nabla \log \Lambda_{K}^{(n)}=0[22-24]$. (The derivatives are taken with respect to the Lagrangian coordinates $\boldsymbol{X}$.)

The first-order perturbative solution for the coefficient of stretching $\eta^{(n)}$ is compared to numerical results in Fig. 2, and similarly for the eigenvector $\hat{\mathbf{s}}_{K}^{(n)}$ in Fig. 3 . Unlike the perturbed coefficients of stretching, which eventually diverge from the numerical solution because of the sensitivity to initial conditions, the perturbed eigenvectors converge very rapidly and are always close to the numerical result.

It will be more convenient to express the metric tensor in terms of its eigenvalues and eigenvectors. The metric tensor (2.5) can be written in terms of the coefficients of expansion and characteristic directions as

$$
g^{(n)}=\left[\Lambda^{(n)}\right]^{2} \hat{\mathbf{u}}^{(n)} \hat{\mathbf{u}}^{(n)}+\left[\Lambda^{(n)}\right]^{-2} \hat{\mathbf{s}}^{(n)} \hat{\mathbf{s}}^{(n)} .
$$

To leading order in $K$, we have

$$
g_{K}^{(n)}=\Lambda^{2 n} \hat{\mathbf{u}} \hat{\mathbf{u}}+\Lambda^{-2 n} \hat{\mathbf{s}} \hat{\mathbf{s}}+2 K \eta^{(n)}\left(\Lambda^{2 n} \hat{\mathbf{u}} \hat{\mathbf{u}}-\Lambda^{-2 n} \hat{\mathbf{s}} \hat{\mathbf{s}}\right)+K \zeta^{(n)}\left(\Lambda^{2 n}-\Lambda^{-2 n}\right)(\hat{\mathbf{u}} \hat{\mathbf{s}}+\hat{\mathbf{s}} \hat{\mathbf{u}})
$$




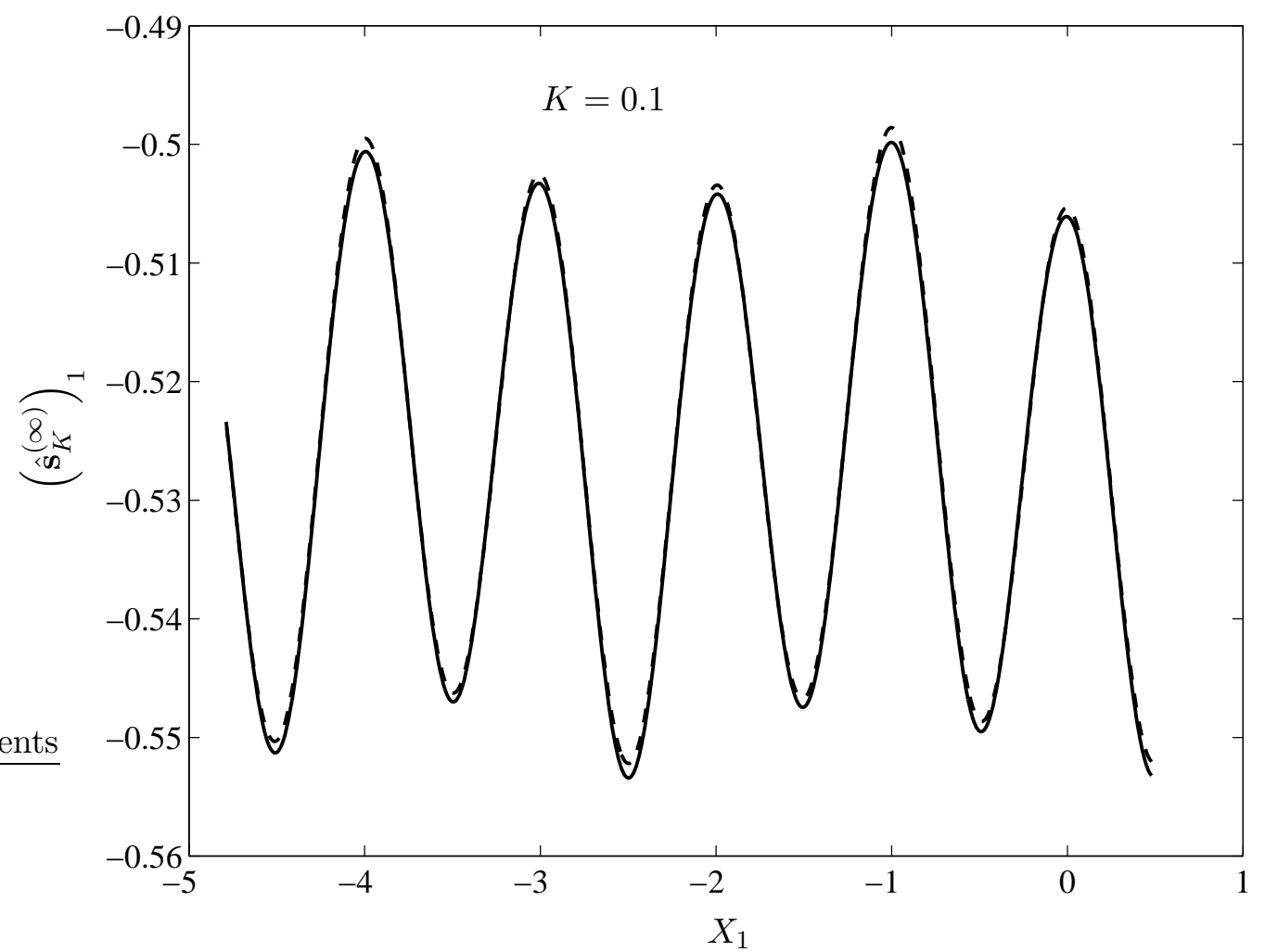

FIG. 3: Numerical solution (solid) and first-order solution (dashed) for the first component of the asymptotic eigenvector $\hat{\mathbf{s}}_{K}^{(\infty)}$ with $K=0.1$, using the asymptotic result (2.17) in (2.13). The error is of order $K^{2}$.

The only dependence on $\boldsymbol{X}$ in (2.19) is contained in $\eta^{(n)}$ and $\zeta^{(n)}$.

\section{ADVECTION AND DIFFUSION}

Having derived the coefficients of expansion and characteristic directions of stretching (to leading order in $K$ ), we can now solve the advection-diffusion equation in Lagrangian coordinates. We will first discuss the case for an incompressible flow, and then make the transition to a volume-preserving map.

The advection-diffusion equation for the concentration of a scalar, $\Theta(\boldsymbol{x}, t)$, advected by an incompressible velocity field $\boldsymbol{v}$ is

$$
\partial_{t} \Theta+\boldsymbol{v} \cdot \partial_{\boldsymbol{x}} \Theta=\kappa \partial_{\boldsymbol{x}}^{2} \Theta
$$

where $\kappa$ is the diffusion coefficient. We define the transformation $\boldsymbol{x}(\boldsymbol{X}, t)$ from Lagrangian coordinates $\boldsymbol{X}$ to Eulerian coordinates $\boldsymbol{x}$ by

$$
\dot{\boldsymbol{x}}=\boldsymbol{v}(\boldsymbol{x}, t), \quad \boldsymbol{x}(\boldsymbol{X}, 0)=\boldsymbol{X},
$$

where the overdot denotes a time derivative at fixed $\boldsymbol{X}$. We can then transform (3.1) to Lagrangian coordinates $\boldsymbol{X}$ [19, 22],

$$
\dot{\Theta}=\partial_{\boldsymbol{X}} \cdot\left(\mathbb{D} \cdot \partial_{\boldsymbol{X}} \Theta\right)
$$


where we reused the same symbol for $\Theta(\boldsymbol{X}, t)$. The anisotropic, nonhomogeneous, timedependent diffusion tensor $\mathbb{D}$ is

$$
\mathbb{D}:=\kappa g^{-1}, \quad g_{p q}:=\sum_{m} \frac{\partial x_{m}}{\partial X_{p}} \frac{\partial x_{m}}{\partial X_{q}},
$$

where $g$ is the metric tensor encountered in Section II. By construction, the advection term has disappeared from (3.3). The flow enters (3.3) indirectly through the metric tensor in (3.4), reflecting the enhancement to diffusion due to the deformation of fluid elements [19, $22]$.

We now make the leap from a flow to a map: because the velocity field does not enter (3.3) directly, we may regard the time dependence in $\mathbb{D}$ as given by a map rather than a flow, and use the metric (2.5) in the diffusion tensor $\mathbb{D}[27]$. We also write $\Theta^{(n)}(\boldsymbol{X})$ for $\Theta(\boldsymbol{X}, t)$, where $n$ denotes the $n$th iterate of the map.

Since our map is defined on the torus, we can expand $\Theta^{(n)}(\boldsymbol{X})$ in Fourier components $\widehat{\Theta}_{\boldsymbol{k}}^{(n)}$; the resulting map, obtained by first Fourier transforming and then solving (3.3), is

$$
\widehat{\Theta}_{k}^{(n)}=\sum_{\ell} \exp \left(\mathcal{G}^{(n)}\right)_{\boldsymbol{k} \ell} \widehat{\Theta}_{\ell}^{(n-1)}
$$

where

$$
\mathcal{G}_{\boldsymbol{k} \boldsymbol{\ell}}^{(n)}=-4 \pi^{2} T \int_{\mathcal{T}^{2}}\left(\boldsymbol{k} \cdot \mathbb{D}^{(n)} \cdot \boldsymbol{\ell}\right) \mathrm{e}^{-2 \pi \mathrm{i}(\boldsymbol{k}-\ell) \cdot \boldsymbol{x}} \mathrm{d}^{2} x,
$$

with $T$ the period of the map. This is an exact result, but the great difficulty lies in calculating the exponential of $\mathcal{G}^{(n)}$. Again, we shall accomplish this perturbatively.

For the torus map introduced in Section II, from Eq. (2.19) we obtain $\left[g_{K}^{(n)}\right]^{-1}=\Lambda^{2 n} \hat{\mathbf{s}} \hat{\mathbf{s}}+\Lambda^{-2 n} \hat{\mathbf{u}} \hat{\mathbf{u}}+2 K \eta^{(n)}\left(\Lambda^{2 n} \hat{\mathbf{s}} \hat{\mathbf{s}}-\Lambda^{-2 n} \hat{\mathbf{u}} \hat{\mathbf{u}}\right)-K \zeta^{(n)}\left(\Lambda^{2 n}-\Lambda^{-2 n}\right)(\hat{\mathbf{u}} \hat{\mathbf{s}}+\hat{\mathbf{s}} \hat{\mathbf{u}})$,

to leading order in $K$, where the only functions of $\boldsymbol{X}$ are $\eta^{(n)}$ and $\zeta^{(n)}$. Inserting (3.7) into (3.6), we find

$$
\mathcal{G}_{\boldsymbol{k} \boldsymbol{\ell}}^{(n)}=A_{\boldsymbol{k} \boldsymbol{\ell}}^{(n)}+K B_{\boldsymbol{k} \boldsymbol{l}}^{(n)}
$$

where

$$
A_{\boldsymbol{k} \boldsymbol{\ell}}^{(n)}:=-\epsilon\left(\Lambda^{2 n} k_{\mathrm{s}}^{2}+\Lambda^{-2 n} k_{\mathrm{u}}^{2}\right) \delta_{\boldsymbol{k} \boldsymbol{\ell}}
$$

and

$$
B_{\boldsymbol{k} \ell}^{(n)}:=-\epsilon\left(2\left(\Lambda^{2 n} k_{\mathrm{s}} \ell_{\mathrm{s}}-\Lambda^{-2 n} k_{\mathrm{u}} \ell_{\mathrm{u}}\right) \eta_{\boldsymbol{k} \boldsymbol{\ell}}^{(n)}-\left(k_{\mathrm{u}} \ell_{\mathrm{s}}+k_{\mathrm{s}} \ell_{\mathrm{u}}\right)\left(\zeta_{+}^{(n)} \boldsymbol{k} \boldsymbol{l}+\zeta_{-}^{(n)} \boldsymbol{k} \ell\right)\right) .
$$

Here we have defined

$$
\epsilon:=4 \pi^{2} \kappa T
$$

to agree with the notation in Ref. [8], as well as

$$
k_{\mathrm{u}}:=\boldsymbol{k} \cdot \hat{\mathbf{u}}, \quad k_{\mathrm{s}}:=\boldsymbol{k} \cdot \hat{\mathbf{s}},
$$

and similarly for $\ell_{\mathrm{u}}$ and $\ell_{\mathrm{s}}$.

Upon making use of the Fourier-transformed (2.12), (2.15), and (2.16) in (3.10), we find

$$
B_{\boldsymbol{k} \ell}^{(n)}=-\frac{1}{2} \epsilon \sum_{m=0}^{n-1} \mathcal{B}_{\boldsymbol{k} \ell}^{n m}\left(\delta_{\boldsymbol{k}, \boldsymbol{\ell}+\hat{\mathbf{e}}_{1} \cdot \mathbb{M}^{m}}+\delta_{\left.\boldsymbol{k}, \boldsymbol{\ell}-\hat{\mathbf{e}}_{1} \cdot \mathbb{M}^{m}\right)}\right)
$$


where $\hat{\mathbf{e}}_{1}$ is a unit vector in the $x_{1}$ direction, and

$$
\mathcal{B}_{k \ell}^{n m}:=\sin 2 \theta\left(\Lambda^{2 n} k_{\mathrm{s}} \ell_{\mathrm{s}}-\Lambda^{-2 n} k_{\mathrm{u}} \ell_{\mathrm{u}}\right)+\left(k_{\mathrm{u}} \ell_{\mathrm{s}}+k_{\mathrm{s}} \ell_{\mathrm{u}}\right)\left(\Lambda^{2(n-m)} \sin ^{2} \theta-\Lambda^{-2(n-m)} \cos ^{2} \theta\right) .
$$

To obtain the full solution, we must now exponentiate (3.8) to give the transfer matrix in (3.5). Fortunately, for $A$ diagonal there is a simple expansion,

$$
\left[\exp \left(A^{(n)}+K B^{(n)}\right)\right]_{\boldsymbol{k} \ell}=\mathrm{e}^{A_{\boldsymbol{k} k}^{(n)}} \delta_{\boldsymbol{k} \ell}+K E_{\boldsymbol{k} \ell}^{(n)} ; \quad E_{\boldsymbol{k} \ell}^{(n)}:=B_{\boldsymbol{k} \ell}^{(n)} \frac{\mathrm{e}^{A_{k \boldsymbol{k}}^{(n)}-\mathrm{e}^{A_{\ell \ell}^{(n)}}}}{A_{\boldsymbol{k} \boldsymbol{k}}^{(n)}-A_{\ell \ell}^{(n)}},
$$

valid to first order in $K$. We say fortunately because without such a formula it is very difficult to compute this matrix exponential - even numerically - due to the large size of the matrices (i.e., infinite) and their magnitude (i.e., growing exponentially in time).

The $\Lambda^{2 n}$ term in $A_{k k}^{(n)}$ seems to imply that $\widehat{\Theta}_{k}^{(n)}$ decays superexponentially fast as $\exp \left(-\epsilon \Lambda^{2 n} k_{\mathrm{s}}^{2}\right)$. From Eulerian considerations [8], we know that for $K \neq 0$ the decay is actually exponential after a short superexponential transient. This is because the $E^{(n)}$ term must be taken into account: it breaks the diagonality of $\mathcal{G}^{(n)}$, so that given some initial set of wavevectors, the concentration contained in those modes can be transferred elsewhere. In particular, it can transfer concentration to modes aligned with the unstable direction. We will see how this avoids superexponential decay in Section IV.

\section{NUMERICAL RESULTS}

\section{A. The Numerical Method}

At this point, solving (3.5) and (3.15) numerically seems like the only way forward. Clearly, attempting the solve this on a grid in Fourier space is hopeless: very high wavenumber modes are quickly populated so the resolution is exhausted very rapidly. Instead, the procedure we use involves keeping track of a list of excited Fourier modes (i.e., those that are nonzero to machine precision). We now describe this scheme.

First, an initial wavenumber is seeded with some initial concentration. This mode will be damped by the diagonal part of the matrix in (3.15), and will also excite two new modes as seen in (3.13). Repeating this, starting now from three modes, we see that the number of excited modes grows exponentially. Thus it would seem that this procedure is not very advantageous; however, after a few iteration the diffusivity (the diagonal part in (3.15)) will damp most modes because $A_{k k}^{(n)}$ is growing exponentially. Thus the modes that have been damped beyond redemption can be removed from the list. In this manner the number of excited modes eventually reaches a constant, though they consist of ever higher wavenumbers. Thus one can think of a "packet" of modes cascading through Fourier space towards larger wavenumbers. It is this packet that is the Lagrangian analogue of the strange eigenmode in Eulerian space, as we will discuss in Section IV B.

Let us first present some results. Figure 4 shows the decay of the scalar variance for $K=0.001$ and four values of the diffusivity. The agreement with the Eulerian results is excellent for early times, but inevitably breaks down later. (In fact the variance eventually begins to increase, which is forbidden.) The agreement is also worse for smaller diffusivity. Both of these disagreements are a manifestation of the wavenumber dependence of the perturbation in (3.15): for $k$ too large the perturbation becomes large, invalidating 


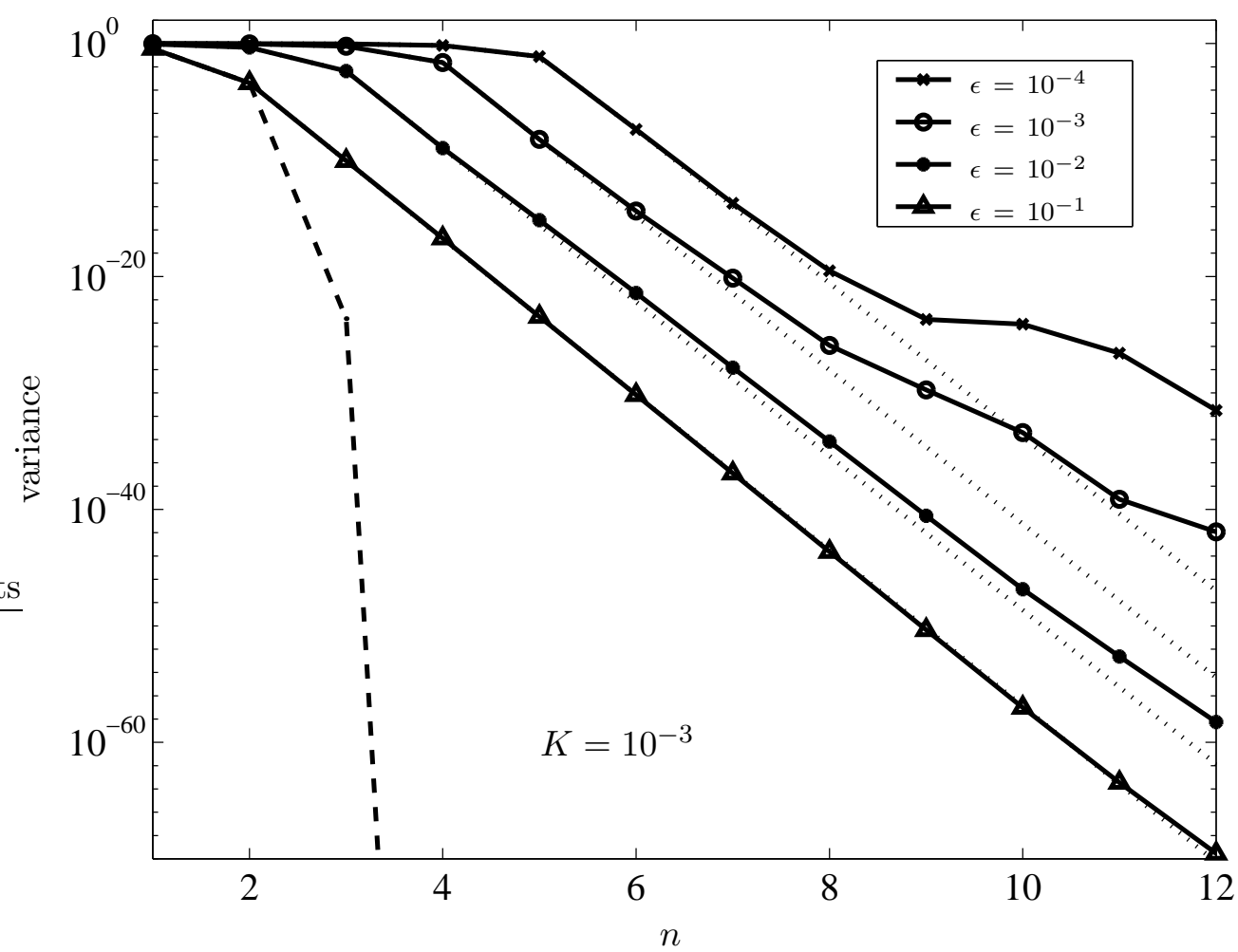

FIG. 4: Decay of the variance for $K=0.001$ and different values of $\epsilon$, compared to the result from Eulerian coordinates (dotted lines). The dashed line shows the exact result for superexponential decay $(K=0)$ for $\epsilon=0.1$.

the approach. Nevertheless, Fig. 4 clearly validates the calculation for times that are not too long.

Another validation is shown in Fig. 5, where we plot the difference between the Eulerian and Lagrangian results as a function of $K$. The difference clearly scales as $K^{-2}$, showing that the two agree at leading order, as required for a first-order asymptotic result.

We now interpret our results in greater detail, and look for a manifestation of the strange eigenmode in Lagrangian coordinates.

\section{B. The Lagrangian Strange Eigenmode}

The mechanism described in Section IV A is similar to that originally introduced (in the context of the kinematic dynamo problem) by Zeldovich et al. [17]: they basically solved the advection-diffusion equation in Lagrangian coordinates for a linear velocity field, and found that in order to avoid rapid superexponential decay one had to restrict attention to a "cone" of wavenumbers that are closely aligned with the unstable manifold of the flow (a similar approach was used later in Refs. [1-3]). The exponential shrinking in time of this "cone of safety" leads to an exponential decay of scalar variance at a rate given by the Lyapunov exponents.

The problem with that approach is that a linear velocity field offers no possibility of dispersion in Fourier space. The wavenumbers in the cone of safety must have some con- 


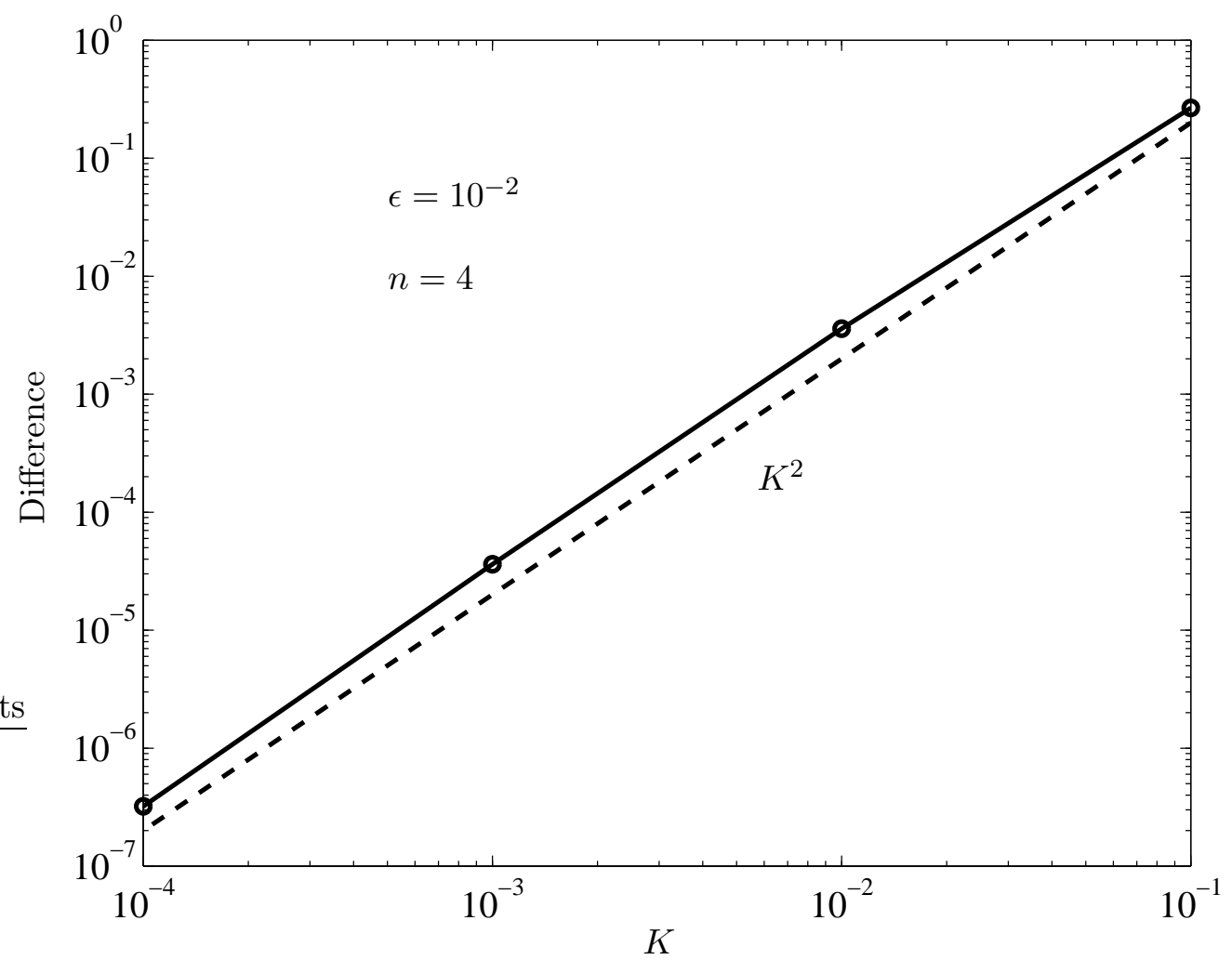

FIG. 5: Relative difference in the variance between the Eulerian and perturbative Lagrangian result as a function of $K$, at the fourth iteration. The dashed line indicates an $K^{2}$ dependence, showing that the two agree to first order in $K$.

centration associated with them initially. What our numerical results show is that if one considers dispersion in Fourier space (of the type allowed by the $E^{(n)}$ term in (3.15)) then it is possible for concentration to be moved inside the cone from elsewhere. The Lagrangian equivalent of the strange eigenmode must live within the cone of safety, otherwise it would decay away superexponentially. But unlike Ref. [17] the decay rate in the present case is not determined by the shrinking of the cone: it is set by how much variance gets transferred into the cone at each iteration.

Figure 6 shows a plot of the power spectrum of concentration. The magnitude of the concentration $\left(\log _{10}\right)$ is plotted vs the magnitude of the wavenumber normalized by $\|\boldsymbol{k}\|_{\max }$ (its maximum value), which is proportional to $\Lambda^{n}$. The concentration is normalized at each iteration such that the mode with largest concentration has unit magnitude. The iterations plotted are $n=6,7,8,9,10,11$ (circles) and $n=12$ (black dots). Most of the circles appear as large black dots, because all these points lie on top of each other. Hence, the concentration is in an eigenstate, given that the wavenumber has been rescaled by a factor proportional to $\Lambda^{n}$ (i.e., such that the dominant peak is at unit rescaled wavenumber). This is what we interpret as the Lagrangian equivalent of the strange eigenmode (a good name might be "stretched eigenmode" in Fourier space).

Some points in Fig. 6 exhibit a decay with iteration number (appearing as columns of circles, with higher iteration numbers lower on the graph): they belong to a more rapidlydecaying eigenfunction. Note that the peaks do not sharpen with iteration number, but 


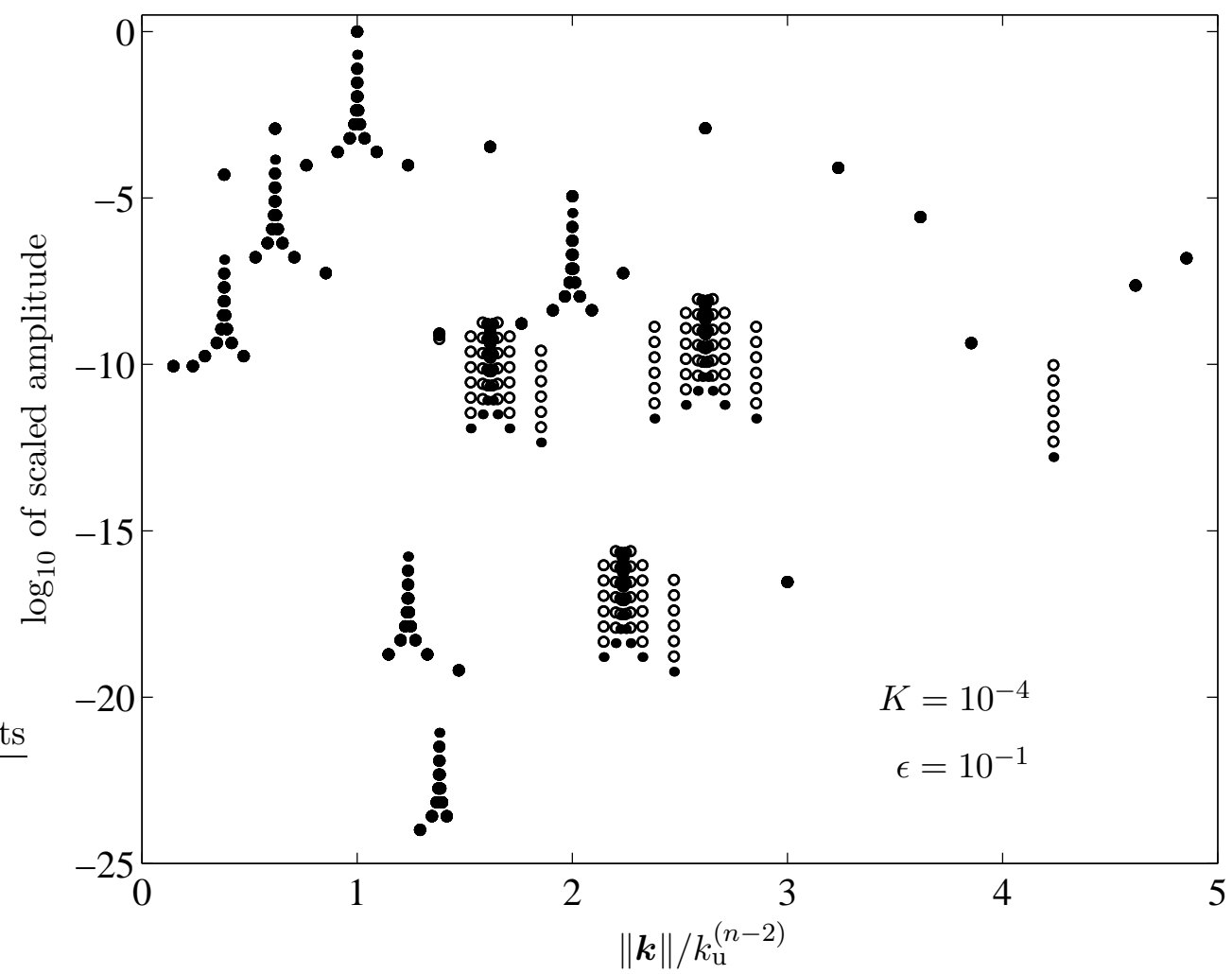

FIG. 6: The power spectrum of the Lagrangian strange eigenmode for $n=6, \ldots, 11$ (circles) and $n=12$ (black dots). The large black dots are points that are the same for all iterations (after rescaling): this is the dominant strange eigenmode in Lagrangian coordinates. Both axes have been rescaled such that the dominant peak has unit amplitude and wavenumber $\left(k_{\mathrm{u}}^{(n-2)}\right.$ is defined in Eq. (A1)). The hollow circles are due to an admixture of another, faster-decaying eigenfunction.

more points are added to some of the tails. The eigenfunction appears extremely rough and discontinuous, though the peaks are indicative of some underlying continuum behavior. The seemingly isolated points actually tend to line up with a peak far below. Finally, note that the relative height (but not position or shape) of the peaks depends on $K$ : the whole shape is stretched vertically as $K$ is made smaller. This is because the term proportional to $K$ controls the transfer of concentration "vertically" (with respect to Fig. 6) in the eigenmode at each iteration.

\section{DISCUSSION}

The Lagrangian strange eigenmode has some intriguing features: (i) It is rescaled exponentially in time, in order to remain within the cone of safety (so it is not a true eigenmode); (ii) Its power spectrum is very discontinuous, in sharp contrast to its Eulerian counterpart [8]; (iii) Its decay rate is set by how much concentration is moved into the "new" cone of safety at each iteration (since the cone is shrinking). In the Appendix we present an analytic result for a two-mode system which gives a simplified representation of the cone of safety.

In the map analyzed here the exponential time-rescaling gives a proper eigenmode, since only the constant stretching is important at leading order. In a generic map the stretching 
is a strong function of space, so the necessary time-rescaling would be position-dependent.

It is hard to see how the Lagrangian approach presented here could be used in more realistic problems: perturbation theory was used extensively (which would not be applicable in most real situations), both for computing the finite-time Lyapunov exponents and the matrix exponential (3.15). We believe the approach is instructive nonetheless, giving as it does a picture of the strange eigenmode in Lagrangian coordinates.

Our approach does not yield much information about the long-time behavior of the decay. There is currently a debate as to whether the mechanism presented in Refs. [1-3] gives a lower bound on the decay rate $[25,26]$. Our perturbation expansion breaks down before this question can be answered.

\section{Acknowledgments}

The author wishes to thank Steve Childress for stimulating discussions.

[1] T. M. Antonsen, Jr., Z. Fan, E. Ott, and E. Garcia-Lopez, "The role of chaotic orbits in the determination of power spectra," Phys. Fluids 8, 3094 (1996).

[2] D. T. Son, "Turbulent decay of a passive scalar in the Batchelor limit: Exact results from a quantum-mechanical approach," Phys. Rev. E 59, R3811 (1999).

[3] E. Balkovsky and A. Fouxon, "Universal long-time properties of Lagrangian statistics in the Batchelor regime and their application to the passive scalar problem," Phys. Rev. E 60, 4164 (1999).

[4] D. R. Fereday, P. H. Haynes, A. Wonhas, and J. C. Vassilicos, "Scalar variance decay in chaotic advection and Batchelor-regime turbulence," Phys. Rev. E 65, 035301(R) (2002).

[5] A. Wonhas and J. C. Vassilicos, "Mixing in fully chaotic flows," Phys. Rev. E 66, 051205 (2002).

[6] A. Pikovsky and O. Popovych, "Persistent patterns in deterministic mixing flows," Europhys. Lett. 61, 625 (2003).

[7] R. T. Pierrehumbert, "Tracer microstructure in the large-eddy dominated regime," Chaos Solitons Fractals 4, 1091 (1994).

[8] J.-L. Thiffeault and S. Childress, "Chaotic mixing in a torus map," Chaos 13, 502 (2003).

[9] W. Liu and G. Haller, "Strange eigenmodes and decay of variance in the mixing of diffusive tracers," Physica D 188, 1 (2004).

[10] J. Sukhatme and R. T. Pierrehumbert, "Decay of passive scalars under the action of single scale smooth velocity fields in bounded two-dimensional domains: From non-self-similar probability distribution functions to self-similar eigenmodes," Phys. Rev. E 66, 056032 (2002).

[11] S. Childress and A. D. Gilbert, Stretch, Twist, Fold: The Fast Dynamo (Springer-Verlag, Berlin, 1995).

[12] M. Pollicott, "On the rate of mixing of Axiom A flows," Invent. Math. 81, 413 (1981).

[13] M. Pollicott, "Meromorphic extensions of generalised zeta functions," Invent. Math. 85, 147 (1986).

[14] D. Ruelle, "Resonances of chaotic dynamical systems," Phys. Rev. Lett. 56, 405 (1986). 
[15] D. Rothstein, E. Henry, and J. P. Gollub, "Persistent patterns in transient chaotic fluid mixing," Nature 401, 770 (1999).

[16] G. A. Voth, T. C. Saint, G. Dobler, and J. P. Gollub, "Mixing rates and symmetry breaking in two-dimensional chaotic flow," Phys. Fluids 15, 2560 (2003).

[17] Y. B. Zeldovich, A. A. Ruzmaikin, S. A. Molchanov, and D. D. Sokoloff, "Kinematic dynamo problem in a linear velocity field," J. Fluid Mech. 144, 1 (1984).

[18] V. I. Arnold, Mathematical Methods of Classical Mechanics, 2nd ed. (Springer-Verlag, New York, 1989).

[19] J.-L. Thiffeault, "Advection-diffusion in Lagrangian coordinates," Phys. Lett. A 309, 415 (2003).

[20] T. Kato, Perturbation Theory for Linear Operators (Springer-Verlag, Berlin, 1980).

[21] E. Merzbacher, Quantum Mechanics (John Wiley \& Sons, New York, 1970).

[22] X. Z. Tang and A. H. Boozer, "Finite time Lyapunov exponent and advection-diffusion equation," Physica D 95, 283 (1996).

[23] J.-L. Thiffeault and A. H. Boozer, "Geometrical constraints on finite-time Lyapunov exponents in two and three dimensions," Chaos 11, 16 (2001).

[24] J.-L. Thiffeault, "Derivatives and constraints in chaotic flows: Asymptotic behaviour and a numerical method," Physica D 172, 139 (2002).

[25] E. Ott, private communication.

[26] D. R. Fereday and P. H. Haynes, "Scalar decay in two-dimensional chaotic advection and Batchelor-regime turbulence," preprint (2003).

[27] It is not possible to simply invoke an incompressible flow corresponding to a map: in general there is no incompressible flow of the same dimension whose trajectories agree with a given map. Formally, however, the replacement of the metric tensor by one corresponding to a map is well-defined mathematically.

\section{APPENDIX A: THE TWO-MODE SOLUTION}

Though we have not found a general method of solution of (3.5) with the exponential given by (3.15), there is at least an approximate solution available that illustrates the broad features of a full solution. It also shows how the decay rate of the variance can become independent of the diffusivity in the Lagrangian viewpoint, as in Ref. [17].

The method is based on defining a class of "aligned" wavenumbers (i.e., that live inside the cone of safety), and retaining only two of these modes. These wavenumbers $\boldsymbol{k}^{(p)}$ are defined by

$$
k_{\mathrm{u}}^{(p)}=\Lambda^{p} \sin \theta, \quad k_{\mathrm{s}}^{(p)}=\Lambda^{-p} \cos \theta,
$$

that is, $\boldsymbol{k}^{(p)}=\mathbb{M}^{p} \cdot \boldsymbol{k}_{0}$, where $\boldsymbol{k}_{0}$ is any initial wavenumber for large enough $p$. Then $\boldsymbol{k}$ satisfies

$$
\boldsymbol{k}^{(p)}-\boldsymbol{k}^{(p-1)}=\hat{\mathbf{e}}_{1} \cdot \mathbb{M}^{p-1},
$$

so that with the choice $\boldsymbol{k}=\boldsymbol{k}^{(p)}, \boldsymbol{\ell}=\boldsymbol{k}^{(p-1)}$, the first Kronecker delta in (3.13) is unity for $m=p-1$.

At the $n$th iteration, assume that only two wavenumbers are important: $\boldsymbol{k}^{(n-d)}$ and $\boldsymbol{k}^{(n-d-1)}$. The number $d$ is a "lag" from the current iteration and will be adjusted later. 
Define

$$
\mathcal{A}_{d}:=\exp A_{\boldsymbol{k}^{(n-d)}}^{(n)} \boldsymbol{k}^{(n-d)}=\exp \left(-\epsilon\left(\Lambda^{2 d} \cos ^{2} \theta+\Lambda^{-2 d} \sin ^{2} \theta\right)\right)
$$

which is independent of $n$, since we have defined $p$ relative to the current iteration of the map. It can be shown that then the coupling from $\boldsymbol{k}^{(n-d-1)}$ to $\boldsymbol{k}^{(n-d)}$ takes the simple form

$$
\mathcal{E}_{d}:=K E_{\boldsymbol{k}^{(n-d)}}^{(n)} \boldsymbol{k}^{(n-d-1)}=-\frac{1}{2} K\left(\mathcal{A}_{d}-\mathcal{A}_{d-1}\right) .
$$

To recapitulate: at the $n$th iteration, the mode $\boldsymbol{k}^{(n-d)}$ is mapped to itself with coupling amplitude $\mathcal{A}_{d}$, and $\boldsymbol{k}^{(n-d-1)}$ is mapped to $\boldsymbol{k}^{(n-d)}$ with amplitude $\mathcal{E}_{d}$. It is easy to show that in this two-mode situation the decay rate is determined by the magnitude of $\varepsilon_{d}$. All that remains is to find $d$.

The "lag" $d$ is obtained by maximizing $\mathcal{E}_{d}$ over $d ; d$ has to be large enough that $\Lambda^{2 d}$ overcomes the tiny diffusivity in (A3) - so the two $\mathcal{A}$ terms don't cancel in (A4) - but not so large that $\mathcal{A}_{d}$ is damped. We are thus justified in approximating $\mathcal{A}_{d} \simeq \exp \left(-\epsilon \Lambda^{2 d} \cos ^{2} \theta\right)$ (the other term in (A3) is smaller by a factor $\Lambda^{-4 d}$, which is small even for $d=1$ ). We then have

$$
\mathcal{E}_{d}=\frac{1}{2} K\left(\Upsilon-\Upsilon^{\Lambda^{2}}\right), \quad \Upsilon:=\mathcal{A}_{d-1},
$$

since $\mathcal{A}_{d}=\left(\mathcal{A}_{d-1}\right)^{\Lambda^{2}}$. This is easily extremized over $\Upsilon$ : the maximum $\left|\mathcal{E}_{d}\right|$ is achieved for $\Upsilon=\Lambda^{-2 /\left(\Lambda^{2}-1\right)} \simeq 0.7198$, for which $\left|\mathcal{E}_{d}\right| \simeq 0.3074 K$. The lag is then given by solving for $d$ in terms of the extremizing $\Upsilon$,

$$
d=1+\frac{1}{2 \log \Lambda} \log \left(\frac{\log \Upsilon^{-1}}{\epsilon \cos ^{2} \theta}\right) \simeq 0.5902+0.5195 \log \epsilon^{-1},
$$

which scales logarithmically with the diffusivity. Note that the decay rate is now completely independent of the actual value of the diffusivity: the lag adjusts itself to compensate, introducing a separation of scale between the dominant wavenumber $\boldsymbol{k}^{(n-d)}$ and the largest wavenumber in the system, $\boldsymbol{k}^{(n)}$.

The actual decay rate as $\epsilon \rightarrow 0$ (from the Eulerian solution in [8]) is $0.5 K$ for small $K$, compared to the two-mode Lagrangian solution $0.3074 K$. Thus, most of the important behavior is captured by the two-mode solution. The two modes can be seen in the spectrum of the strange eigenmode in Fig. 6: the dominant peak at $\|\boldsymbol{k}\| / k_{\mathrm{u}}^{(n-2)}=1$ is $\boldsymbol{k}^{(n-d)}(d=2$ in this case), and the peak at $\|\boldsymbol{k}\| / k_{\mathrm{u}}^{(n-3)}=\Lambda^{-1} \simeq 0.3820$ is $\boldsymbol{k}^{(n-d-1)}$. The other peaks are modes that could be included to get a more accurate expression for the decay rate.

The two-mode solution also nicely illustrates the idea of the cone of safety: both modes are always inside it, and because the cone is shrinking by a factor $\Lambda^{-1}$ at each iteration then the modes have to follow suit. The key difference with [17] is that here the concentration in the modes is mapped from one cone to another at each iteration, and is not part of the initial condition. 